\title{
Postoperative Neuropsychological Outcome in Patients with Mesial Temporal Lobe Epilepsy in Argentina
}

\author{
Silvia Oddo, ${ }^{1,2,3,4}$ Patricia Solis, ${ }^{1,2,3}$ Damian Consalvo, ${ }^{1,2,3}$ Eduardo Seoane, ${ }^{1}$ \\ Brenda Giagante, ${ }^{1,2,3}$ Luciana D'Alessio, ${ }^{1,2,3}$ and Silvia Kochen ${ }^{1,2,3,4}$ \\ ${ }^{1}$ Epilepsy Center, Neurology Division, Ramos Mejia Hospital, Urquiza 609, Buenos Aires, PC C1221ADC, Argentina \\ ${ }^{2}$ Neuroscience and Cellular Biology Institute "Prof. Dr. Eduardo De Robertis", Medicine Faculty, Buenos Aires University, Paraguay \\ 2155, Buenos Aires City, PC C1121ABG, Argentina \\ ${ }^{3}$ Research Center of Pharmacobiology and Botanic, CEFYBO, Buenos Aires University, Paraguay 2155, Buenos Aires City, \\ PC C1121ABG, Argentina \\ ${ }^{4}$ National Council of Scientific and Technology Research (CONICET), Buenos Aires, Argentina
}

Correspondence should be addressed to Silvia Oddo, silviaoddo@hotmail.com

Received 11 April 2011; Revised 10 August 2011; Accepted 8 October 2011

Academic Editor: Seyed M. Mirsattari

Copyright (c) 2012 Silvia Oddo et al. This is an open access article distributed under the Creative Commons Attribution License, which permits unrestricted use, distribution, and reproduction in any medium, provided the original work is properly cited.

\begin{abstract}
The aim of the present study is to compare pre- and postsurgical neuropsychological outcome in individuals suffering from mesial temporal lobe epilepsy (mTLE), in order to evaluate prognosis. The selected thirty-five patients had medically mTLE and had undergone an anterior temporal lobectomy (ATL). Neuropsychological evaluation was performed in three different stages: before ATL, 6 months after resection, and a year afterwards. Neuropsychological protocol evaluated attention, verbal memory, visual memory, executive function, language, intelligence, and handedness. There was a significant improvement $(P=0.030)$ in the group with visual memory deficit after surgery, whereas no changes were observed across patients with verbal memory deficit. No changes were observed in language after surgery. Executive function showed significant improvement 6 months after surgery $(P=0.035)$. Postoperative outcome of cognitive impairments depends on baseline neuropsychological status of the patients with TLE. In our case series, deficits found in patients with mTLE after ATL did not result in a subjective complaint.
\end{abstract}

\section{Introduction}

Neuropsychological evaluation is a very important tool to characterize cognitive aspects in patients with epilepsy and to determine the topography of the epileptogenic zone [1-4].

In a previous study conducted by our group [4], patients with mesial temporal lobe epilepsy (mTLE) [5] displayed a neuropsychological profile characterized by material-specific verbal/visual episodic memory, executive function, and speech impairment. In most cases, memory deficits correlated with the lesions detected by MRI [4]. These findings were similar to those reported by other authors.

A high percentage of patients with TLE syndrome benefit from temporal lobe resection [6]. In the history of epilepsy surgery, neuropsychological evaluation played a major role to understand some aspects of cognition. There are several publications concerning the prognosis of cognitive functions among patients undergoing epilepsy surgery $[3,7,8]$. Verbal memory deficits were observed after left temporal lobectomy (TLY). On the other hand, visual memory deficits were found after right TLY. These findings are similar to those described in published studies conducted among English populations.

The aim of the present study is to compare pre- and postsurgical neuropsychological outcome in individuals suffering from TLE in order to evaluate prognosis across a Spanish-speaking population.

\section{Materials and Methods}

The selected thirty-five patients with mesial temporal sclerosis (MTS) were treated at the Epilepsy Center of the Hospital Ramos Mejía. All the studied patients had MTS on their MRI [4]. 
The type of surgery performed consisted of resection of the anterior temporal lobe, as well as a resection of the anterior mesial temporal structures including the anterior third of the hippocampus (Standard Anterior Temporal Lobectomy, ATL). Lateral temporal neocortex and mesial structures were removed at approximately $3 \mathrm{~cm}$ from the temporal pole within the dominant hemisphere, and up to $4.5 \mathrm{~cm}$ in the nondominant hemisphere.

Fifty one percent of the patients underwent a left TLY and $49 \%(n=17)$ underwent a right TLY.

The neuropsychological protocol used in this study was the same that was previously published by our group. The verbal tests were validated for the Spanish language $[1,4,9]$.

Neuropsychological evaluation was performed in three different stages: before surgery, 6 months after resection, and a year afterwards.

(i) Attention: Forward and Backward Digit Span, WAIS, and Trail Making Test part A.

(ii) Verbal memory: Rey Auditory Verbal Learning Test (RAVLT) and List Learning Test.

(iii) Visual memory: Rey-Osterrieth Complex Figure Test (RCFT).

(iv) Executive function: Wisconsin Card Sorting Test (WCST), Trail Making Test Part B. Verbal Fluency (FAS).

(v) Language: Boston Naming Test (BNT), Token Test (TT).

(vi) Intelligence quotient (IQ).

(vii) Handedness: Edinburgh Questionnaire (EHQ).

Patients who had an IQ below 70 were excluded from the study.

\section{Statistical Analysis}

The results of the neuropsychological evaluation were compared with the normative according to age, sex, and formal education. For each patient the raw values of each data of the cognitive tests were normalized to a $Z$ score. Patients were classified as "normal" when all the tests presented values superior to a $Z$ score -2 (two standard deviation below normal values), or "abnormal" when some of the results were inferior to a $Z$ score -2 .

In a second stage of analysis, we divided results obtained in RAVLT, RCFT, WSCT, FAS, and BNT, into three groups: Baseline with ( $n=35$ patients), 6 months with $(n=35$ patients), and a year from surgery with ( $n=14$ patients), due to a loss of followup in the rest of the patients.

We used raw values. We did not compare them with normative scores. We compared means as follows:

(i) baseline/ 6 months: $n=35$ patients,

(ii) baseline/year: $n=14$ patients,

(iii) 6 months/year: $n=14$ patients. In order to match the number of patients in each group to compare two samples, we decided to form two groups with 14 patients.
TABLE 1: Patients profile.

\begin{tabular}{lll}
\hline Gender (F/M \%) & $45 / 55$ & \\
Age (average years) & $36.1 \pm 8$ & \\
Time of epilepsy evolution & $21.1 \pm 12$ & \\
Education (average years) & $10.6 \pm 4$ & \\
Handedness (R/L) & $94 / 6$ & \\
RTLY/LTLY (\%) & $49 / 51$ & 70 \\
MRI lesion (\%) & HE & 18 \\
& HE plus & 9 \\
\hline
\end{tabular}

HS: Hippocampal Sclerosis. RTLY: Right temporal lobectomy. LTL: Left temporal lobectomy.

Were used ANOVA and Chi-Square tests for the analyses, to compare dependent and independent variables in a sample of normal distribution and to test the independence of two variables together, by presenting data in contingency tables.

\section{Results}

4.1. Patient Demographics. All patients had drug-resistant mTLE.

The average age of the study population was $36.1 \pm 8$ years.

The average evolution time of epilepsy was $21.1 \pm 12$ years.

The average education was $10.6 \pm 4$ years.

Forty-five percent of the study population was women. Ninety-four percent of the study population was right handed, and the remaining $6 \%$ was left handed.

All patients underwent standard ATL.

$70 \%$ of the patients had hippocampal sclerosis (HS), $18 \%$ had HS plus temporal pole dysplasia, $9 \%$ presented a low degree tumor, and $3 \%$ had cavernoma.

Postoperative categorization of the seizures (Engel scale) [10] was $85.7 \%$ Class I, 8.5\% Class II, and 2.8\% Class III. This was observed one year after surgery (see Table 1).

4.2. Neuropsychological Outcome. In the preoperative assessment, $(28.5 \%)$ out of 35 patients showed a normal neuropsychological evaluation, while 25 patients $(71.4 \%)$ showed significant deficit on any of the tasks.

At six months after surgery, there was an increase in the percentage of patients with normal neuropsychological test scores (13 patients, $37.1 \%$ )

A year after the surgery, the neuropsychological evaluation was completed in 14 patients. There was a similar tendency in the outcome observed 6 months afterwards, because 5 patients $(35.7 \%)$ out of 14 patients presented a normal neuropsychological evaluation, where one of them was abnormal at baseline. Nine patients $(64.2 \%)$ out of 14 patients show significant deficits on at least one of the tasks.

4.3. Intelligence Quotient (IQ). The average of the total IQ score prior to surgery was $91.7 \pm 13,(73-125)$. Six months after the surgery, the average of the total IQ score was 
TABLE 2: Memory outcome after anterior temporal lobectomy based on the side of the surgery.

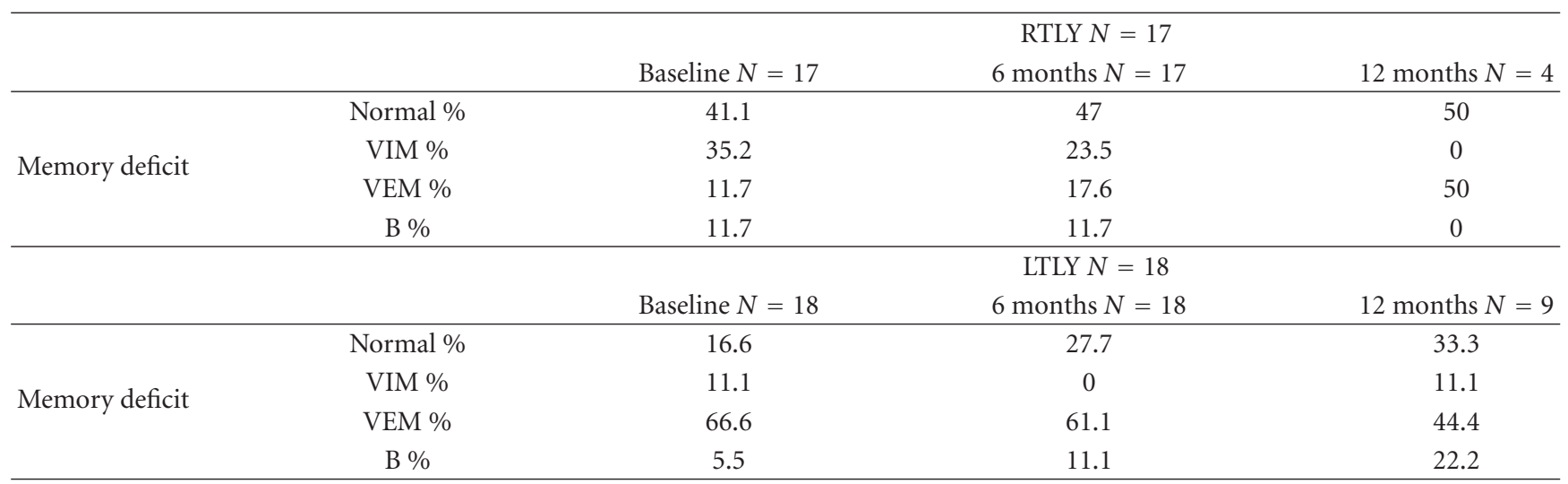

RTLY: Right temporal lobectomy. LTLY: Left temporal lobectomy. VIM: Visual memory. VEM: Verbal memory. B: both memories.

$93.5 \pm 12(74-125)$. A year after the surgery, the average of the total IQ score was $93.7 \pm 12$ (80-125). Therefore, changes were nonsignificant.

4.4. Memory. Baseline evaluation of these 35 patients was included in a previous paper. In that study we found deficits in delayed recall, on RAVLT and RCFT. Therefore, the present analysis was conducted primarily in consideration of those data [4].

Results of memory evaluation for each patient, compared with normative, were as follows.

4.4.1. Left Temporal Lobectomy Group $(n=18)$. At baseline, twelve patients $(66.6 \%)$ presented verbal memory deficits, and two patients $(12 \%)$ had visual memory deficits. One patient $(5.5 \%)$ had both types of memory deficits. Three patients $(16.6 \%)$ did not present any memory deficits.

Six months after surgery, no significant changes were observed.

A year after surgery, the tendency was the same as observed at 6 months after surgery (Table 2).

4.4.2. Right Temporal Lobectomy Group $(n=17)$. At baseline, two patients $(11.7 \%)$ presented with verbal memory deficits, and six patients (35.2\%) had visual memory deficits. Two patients $(11.7 \%)$ had both types of memory deficits. Seven patients $(41.1 \%)$ did not present any memory deficits.

Six months after the surgery, no significant changes were observed.

A year after the surgery, only 4 patients were evaluated (Table 2).

When we compared the raw data means, we found a significant improvement $(P=0.030)$ in the group with visual memory deficits (Table 3 ).

4.5. Language. In the preoperative evaluation, 26 patients $(74.2 \%)$ had speech impairments, while in 9 patients $(25.7 \%)$ language assessment was normal.
Deficits were mostly observed in the naming. The most significant finding was BNT. No significant impairment was observed in the listening comprehension (Token Test).

There were no significant changes 6 months and a year after the surgery (Table 3 ).

4.6. Executive Function. Before surgery, 9 patients (25.8\%) presented with deficits in the Wisconsin Card Sorting Test (WCST). The most frequent alterations were planning and difficulty in mental flexibility to switch strategies, and preservative errors. Verbal production capacity and mental flexibility (FAS) were abnormal in 5 patients (14.2\%).

Six months after surgery, significant improvement was observed in 6 patients $(17 \%)(P=0.035)$ in the evaluation with WCST (Table 2). Verbal production capacity (FAS) did not show significant impairment. 11 patients (31.4\%) showed deficits.

A year after surgery, there were no alterations in the assessment of WCST, while on FAS, all patients improved significantly $(P=0.026)$ (Table 3$)$.

\section{Discussion}

A high percentage of patients with TLE syndrome benefit from temporal lobe resection [6]. In the history of epilepsy surgery, neuropsychological evaluation played a major role to understand some aspects of cognition.

Following the order previously set out in this study, we will proceed to discuss the results of postoperative evaluation.

Regarding IQ, the average of the total score shows no significant improvement 6 months after the surgery and remains stable 12 months afterwards. This has been noted by other authors [11], who point to an overall slight improvement, that might depend on multiple factors such as the absence of seizures, the improvement in the patients' overall quality of life, or simply practice effects.

Memory analysis was performed by discriminating between verbal/visual memory.

Verbal memory deficits were the most frequent findings in patients with left mTLE, while in patients with right 
TABLE 3: Analysis of raw data of RAVLT, RCFT, BNT, WCST, and FAS tests, at baseline, 6 months, and a year after anterior temporal lobectomy.

\begin{tabular}{|c|c|c|c|}
\hline & MEAN & $N$ & $P$ \\
\hline \multicolumn{4}{|l|}{ RALVT } \\
\hline Baseline & 6.8 & 35 & \multirow{2}{*}{0.706} \\
\hline 6 months & 6.55 & 35 & \\
\hline Baseline & 6.57 & 14 & \multirow{2}{*}{0.692} \\
\hline Year & 7.07 & 14 & \\
\hline 6 months & 6.57 & 14 & \multirow{2}{*}{0.692} \\
\hline Year & 7.07 & 14 & \\
\hline \multicolumn{4}{|l|}{ RCFT } \\
\hline Baseline & 14.6 & 35 & \multirow{2}{*}{0.796} \\
\hline 6 months & 14.86 & 35 & \\
\hline Baseline & 15.93 & 14 & \multirow{2}{*}{0.086} \\
\hline Year & 19.07 & 14 & \\
\hline 6 months & 17.5 & 14 & \multirow{2}{*}{0.03} \\
\hline Year & 19.07 & 14 & \\
\hline \multicolumn{4}{|l|}{ BNT } \\
\hline Baseline & 39.51 & 35 & \multirow{2}{*}{0,631} \\
\hline 6 months & 38.8 & 35 & \\
\hline Baseline & 39.21 & 14 & \multirow{2}{*}{0.92} \\
\hline Year & 39.43 & 14 & \\
\hline 6 months & 40.36 & 14 & \multirow{2}{*}{0.753} \\
\hline Year & 39.43 & 14 & \\
\hline \multicolumn{4}{|l|}{ WCST } \\
\hline Baseline & 4.57 & 35 & \multirow{2}{*}{0.268} \\
\hline 6 months & 4.97 & 35 & \\
\hline Baseline & 4.5 & 14 & \multirow{2}{*}{0.378} \\
\hline Year & 5.07 & 14 & \\
\hline 6 months & 4.29 & 14 & \multirow{2}{*}{0.035} \\
\hline Year & 5.07 & 14 & \\
\hline \multicolumn{4}{|l|}{ FAS } \\
\hline Baseline & 23.03 & 35 & \multirow{2}{*}{0.098} \\
\hline 6 months & 18.97 & 35 & \\
\hline Baseline & 25.38 & 14 & \multirow{2}{*}{0.081} \\
\hline Year & 29.77 & 14 & \\
\hline 6 months & 20 & 14 & \multirow{2}{*}{0.026} \\
\hline Year & 29.77 & 14 & \\
\hline
\end{tabular}

BNT: Boston Naming Test, WCST: Wisconsin Card Sorting Test, RAVLT: Rey auditory learning verbal test, FAS: verbal fluency, RFCT: Rey figure copy test.

mTLE, visual memory deficits were frequent observation, but less consistent findings. Memory deficits were most frequently observed in delayed recall of both verbal and visual material. These findings are consistent with lesions in the mesial temporal structures, which seem to be critical for encoding [4].

Significant improvement of visual memory was observed at 6 and 12 months on delayed recall, whereas verbal memory analysis showed no significant changes.

In most cases, patients with normal memory at baseline showed no significant changes after ATL.
After left ATL, patients presented significant verbal memory deficits, whereas after right ATL, visual memory deficit was less consistent, deficits on verbal memory (contralateral to the resection), and remained unchanged or slight improvement [3].

Chelune described the "functional adequacy" phenomenon as the functional adaptation of the tissue to be resected in the ipsilateral temporal lobe to the lesion, and "reserve capacity" as the mnesic capacity of the hippocampus contralateral to the lesion [8]. Thus, those patients who do not present with a memory deficits preoperatively would have an unfavorable prognosis, since "functionally" healthy tissue may be resected, while patients who display memory deficit prior to surgery would have a better prognosis.

As regards language, no significant changes were observed in the postoperative evaluation.

The detection of deficit in the BNT brings about several conclusions. One is the theory that suggests a connection between the hippocampal system and its relationship with the language formation process at the phonological and semantic levels, as well as its relationship with comprehension and production, which reveals a deficit in the retrieval of stored information in the lexical-semantic system [12].

Recently, the same author described the role of the hippocampus in the association of external stimuli with its semantic content. Thus, the damage caused by epilepsy in the lateral and mesial regions of the temporal lobe would affect the neocortical and hippocampal functions involved in the storage and retrieval of such information, leading to a semantic memory deficit $[13,14]$. Another hypothesis holds the existence of atypical areas within the anterior and basal regions of the temporal lobe as a result of neural plasticity mechanisms [15-17].

Thirty percent of our patients displayed deficits in the Executive Function, thus implicating the function of the frontal lobe. This has been noted by other authors, and numerous hypotheses have been suggested. One of the theories holds the possibility that the temporal discharge propagation towards the frontal lobe may interfere with the executive function.

Another plausible theory is that memory deficit may alter the results of the tests assessing frontal function [18-20]. In our population, a significant improvement was observed after surgery, which is consistent with other observations.

\section{Conclusions}

To our knowledge, this is the largest study involving Spanishspeaking patients.

The postoperative prognosis of cognitive impairments depends almost entirely on the patient's prior condition.

In the group of patients with executive function deficits in baseline, there was a significant postsurgical improvement.

Patients who manifested postoperative deficits after ATL did not result in a subjective complaint, and the patients who did not display a cognitive deficit preoperatively remained stable. 
Neuropsychological evaluation turns out to be an important tool in the diagnosis and followup of medically intractable TLE patients after TLY.

\section{References}

[1] R. F. Allegri, M. Drake, and A. Thompson, "Neuropsychological findings in patients with middle temporal lobe epilepsy," Revista de Neurología, vol. 29, no. 12, pp. 1160-1163, 1999 (Spanish).

[2] M. Jones-Gotman, R. J. Zatorre, A. Olivier et al., "Learning and retention of words and designs following excision from medial or lateral temporal lobe structures," Neuropsychologia, vol. 35, no. 7, pp. 963-973, 1997.

[3] T. M. C. Lee, J. T. H. Yip, and M. Jones-Gotman, "Memory deficits after resection from left or right anterior temporal lobe in humans: a meta-analytic review," Epilepsia, vol. 43, no. 3, pp. 283-291, 2002.

[4] S. Oddo, P. Solís, D. Consalvo et al., "Mesial temporal lobe epilepsy and hippocampal sclerosis: cognitive function assessment in Hispanic patients," Epilepsy and Behavior, vol. 4, no. 6, pp. 717-722, 2003.

[5] H. G. Wieser, "Mesial temporal lobe epilepsy with hippocampal sclerosis," Epilepsia, vol. 45, no. 6, pp. 695-714, 2004.

[6] S. Wiebe, W. T. Blume, J. P. Girvin, and M. Eliasziw, "A randomized, controlled trial of surgery for temporal-lobe epilepsy," The New England Journal of Medicine, vol. 345, no. 5, pp. 311-318, 2001.

[7] S. Baxendale, P. Thompson, W. Harkness, and J. Duncan, "Predicting memory decline following epilepsy surgery: a multivariate approach," Epilepsia, vol. 47, no. 11, pp. 1887-1894, 2006.

[8] G. J. Chelune, "Hippocampal adequacy versus functional reserve: predicting memory functions following temporal lobectomy," Archives of Clinical Neuropsychology, vol. 10, no. 5, pp. 413-432, 1995.

[9] R. F. Allegri, C. A. Mangone, A. F. Villavicencio, S. Rymberg, F. E. Taragano, and D. Baumann, "Spanish Boston Naming Test norms," Clinical Neuropsychologist, vol. 11, no. 4, pp. 416-420, 1997.

[10] J. Engel Jr., P. Van Ness, T. Rasmussen, and L. Ojemann, "Outcome with respect to epileptic seizures," in Surgical Treatment of the Epilepsies, J. Engel Jr., Ed., pp. 609-621, Raven Press, New York, NY, USA, 1993.

[11] M. Äikiä, T. Salmenperä, K. Partanen, and R. Kälviäinen, "Verbal memory in newly diagnosed patients and patients with chronic left temporal lobe epilepsy," Epilepsy and Behavior, vol. 2, no. 1, pp. 20-27, 2001.

[12] A. R. Giovagnoli, "Verbal semantic memory in temporal lobe epilepsy," Acta Neurologica Scandinavica, vol. 99, no. 6, pp. 334-339, 1999.

[13] A. R. Giovagnoli, A. Erbetta, F. Villani, and G. Avanzini, "Semantic memory in partial epilepsy: verbal and non-verbal deficits and neuroanatomical relationships," Neuropsychologia, vol. 43, no. 10, pp. 1482-1492, 2005.

[14] A. R. Giovagnoli, "Relation of sorting impairment to hippocampal damage in temporal lobe epilepsy," Neuropsychologia, vol. 39, no. 2, pp. 140-150, 2001.

[15] O. Devinsky, K. Perrine, J. Hirsch, W. McMullen, S. Pacia, and W. Doyle, "Relation of cortical language distribution and cognitive function in surgical epilepsy patients," Epilepsia, vol. 41, no. 4, pp. 400-404, 2000.
[16] O. Devinsky, K. Perrine, R. Llinas, D. J. Luciano, and M. Dogali, "Anterior temporal language areas in patients with early onset of temporal lobe epilepsy," Annals of Neurology, vol. 34, no. 5, pp. 727-732, 1993.

[17] B. P. Hermann and A. R. Wyler, "Effects of anterior temporal lobectomy on language function: a controlled study," Annals of Neurology, vol. 23, no. 6, pp. 585-588, 1988.

[18] M. Drake, R. F. Allegri, and A. Thompson, "Executive cognitive alteration of prefrontal type in patients with mesial temporal lobe epilepsy," Medicina, vol. 60, no. 4, pp. 453-456, 2000 (Spanish).

[19] M. D. Horner, L. A. Flashman, D. Freides, C. M. Epstein, and R. A. E. Bakay, "Temporal lobe epilepsy and performance on the Wisconsin Card Sorting Test," Journal of Clinical and Experimental Neuropsychology, vol. 18, no. 2, pp. 310-313, 1996.

[20] R. C. Martin, S. M. Sawrie, F. G. Gilliam et al., "Wisconsin card sorting performance in patients with temporal lobe epilepsy: clinical and neuroanatomical correlates," Epilepsia, vol. 41, no. 12, pp. 1626-1632, 2000. 


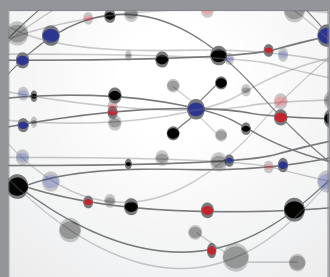

The Scientific World Journal
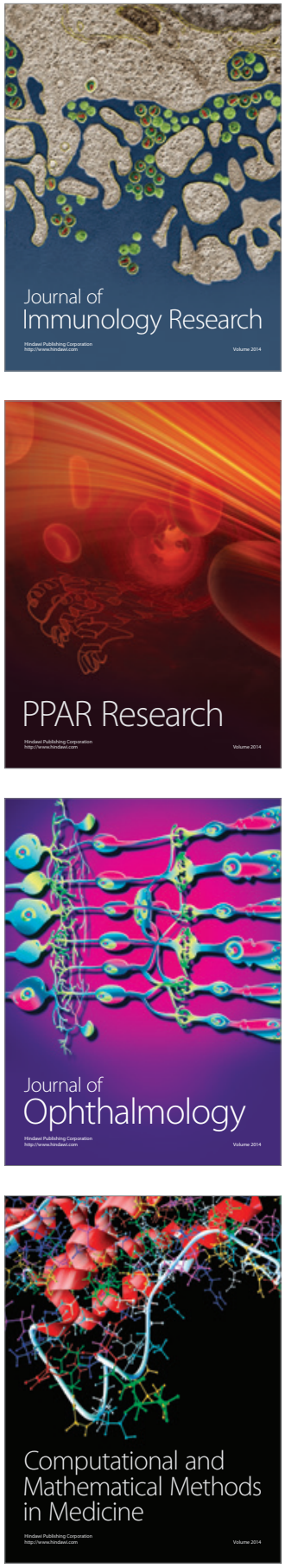

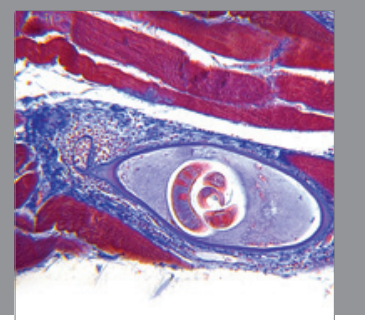

Gastroenterology

Research and Practice
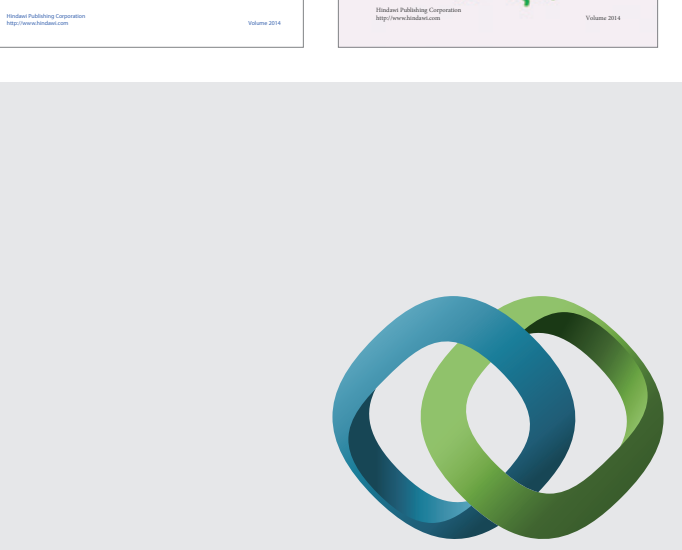

\section{Hindawi}

Submit your manuscripts at

http://www.hindawi.com
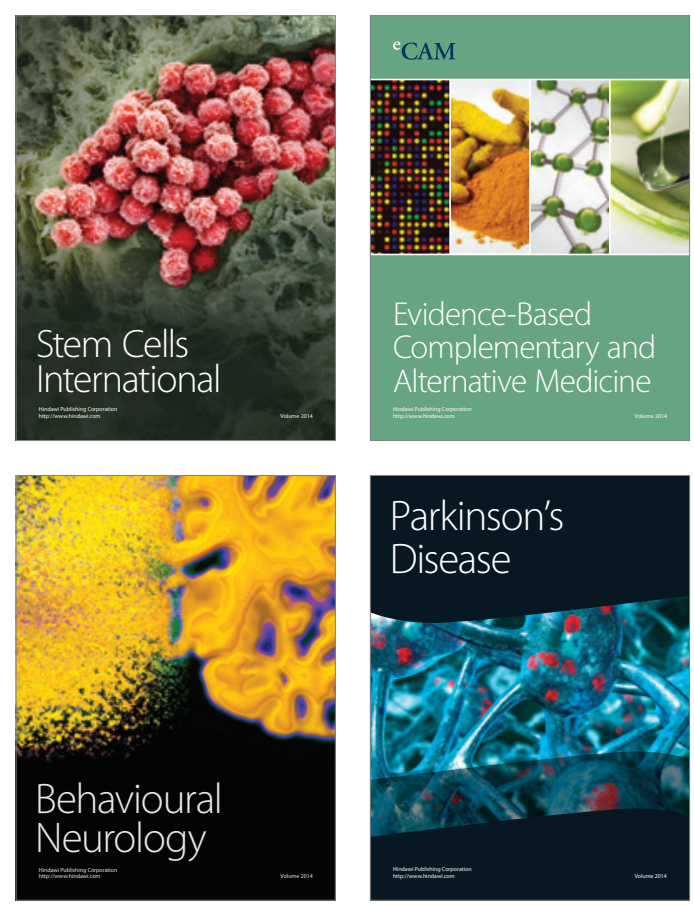

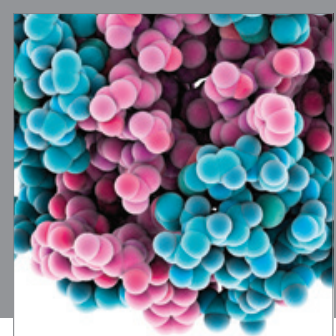

Journal of
Diabetes Research

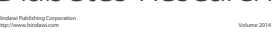

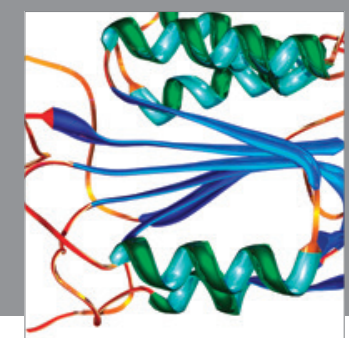

Disease Markers
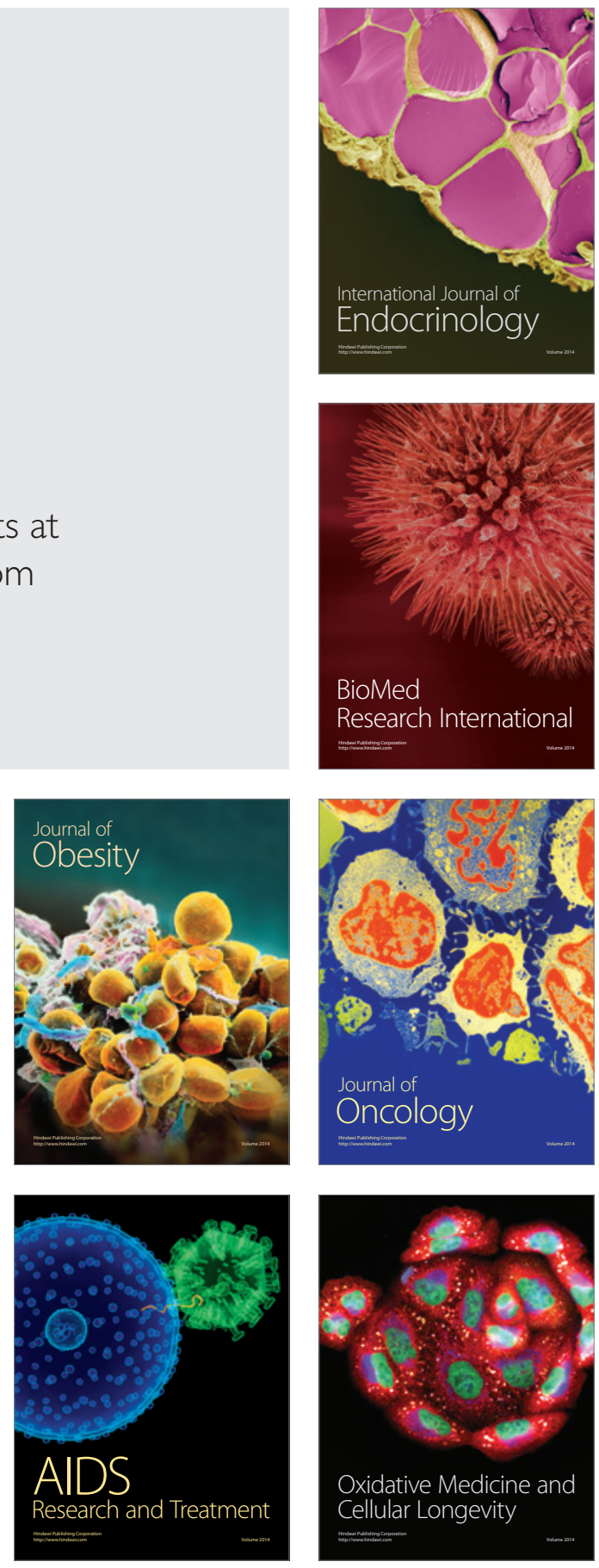